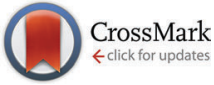

Cite this: Chem. Commun., 2015, 51, 6595

Received 12th February 2015 Accepted 11th March 2015

DOI: $10.1039 / \mathrm{c5cc01334h}$

www.rsc.org/chemcomm

\section{A low molecular weight hydrogel with unusual gel aging $\dagger$}

\author{
Emily R. Draper, Tom O. McDonald and Dave J. Adams*
}

We describe a dipeptide hydrogel with unusual aging characteristics. Over time, a transformation from a turbid gel to a transparent gel occurs which is initiated from the air-water interface. Here, we investigate this transition and discuss the implications of this aging on the bulk properties of the gel.

The self-assembly of low molecular weight gelators (LMWGs) is a useful method to produce well-defined materials with many potential applications, including tissue engineering, drug delivery and organic electronics. ${ }^{1-4}$ Gelation generally occurs when a trigger is applied to a freely dissolved LMWG, leading to the assembly of the molecules into long fibrous structures that then entangle and trap the solvent, forming a gel. These LMWGs are of interest due to the large variety of gelator structures reported, as well as being relatively cheap to produce and simple to scale up.

Generally, the aging of these gels is rarely discussed in the literature. The properties of gels are often reported without stating the age of the material, despite there being examples in the literature showing that gel network formation is not the necessarily the thermodynamic minimum. ${ }^{5-8}$ For example, Smith and $\mathrm{Yu}$ have both reported gel systems that show gel-to-crystal transitions over time, ${ }^{9,10}$ which are attributed to gel formation being a balance between amorphous aggregation and well-ordered crystallisation. In general, gelation is described as the kinetically controlled process, whereas with time the more thermodynamically stable crystallization occurs. This type of gel aging leads to the deformation or weakening of rheological properties of the gel due to disruption of the gel matrix. Other examples of gel aging show Ostwald ripening, where smaller structures fuse together to form larger, more stable structures. ${ }^{1-14}$ Additional LMWGs have been shown to form aggregates when gelation is triggered. Over time these aggregates rearrange or 'self-correct' to form a more

Department of Chemistry, University of Liverpool, Crown Street, Liverpool, L697ZD, UK. E-mail: d.j.adams@liverpool.ac.uk

$\dagger$ Electronic supplementary information (ESI) available: Full synthetic details for the LMWG, photographs of gelation under different conditions, further rheological data. See DOI: 10.1039/c5ec01334h stable and homogeneous network. ${ }^{15-17}$ These gel-to-gel transitions often lead to gels with increased rheological strength. Changes in the gel network may not be a visible process yet could affect the gel properties with time. The stability of gels is important when considering the material for a specific application. ${ }^{18}$ Monitoring the aging of gels may also offer insight into gel network formation. ${ }^{19}$ Here we report a LMWG that has extremely unusual aging properties caused by a gel-to-gel transition.

A solution of the LMWG, 2-thiophene diphenylalanine (Fig. 1a), was dissolved in deionised water at $\mathrm{pH} 11$ using sodium hydroxide to give a transparent solution (Fig. 1b). Glucono- $\delta$ lactone (GdL) was then used to lower the $\mathrm{pH}$ slowly and trigger gelation. ${ }^{20,21}$ We have previously shown that this method allows the formation of uniform gels, and the slow hydrolysis allows the assembly process to be followed. ${ }^{20,22}$ After one hour, the initially transparent solution became turbid. After six hours, an opaque self-supporting gel was formed (Fig. 1c). Unusually, the gel then

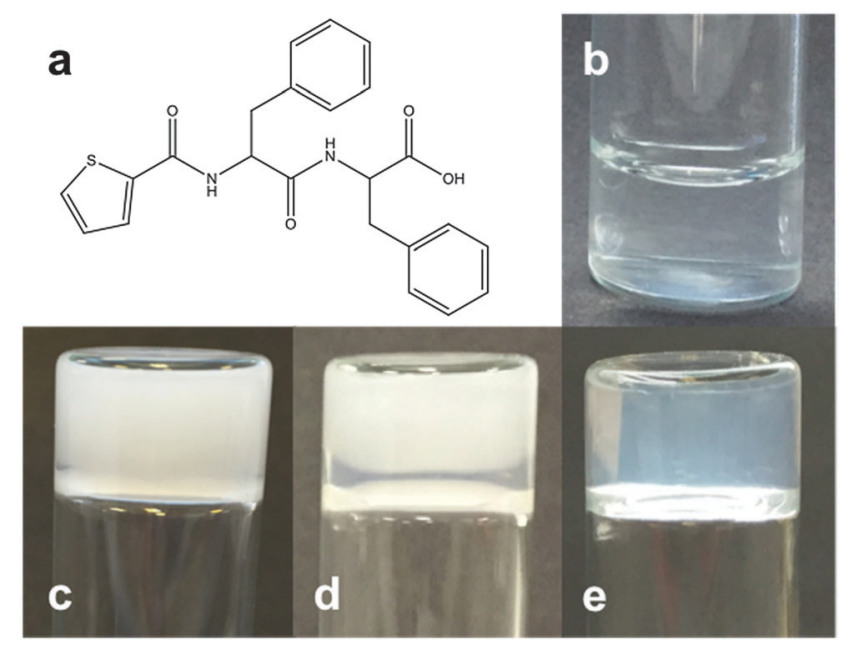

Fig. 1 (a) Structure of the LMWG, 2-thiophene diphenylalanine. (b) LMWG in high $\mathrm{pH}$ water. (c) Turbid gel formed after 8 hours. (d) The gel becoming transparent after 16 hours. (e) A completely transparent gel is formed after 3 days. 
started to become transparent, starting from the air-water interface (Fig. 1d) and was completely transparent after three days (Fig. 1e).

The transparent, aged gel showed significantly stronger rheological properties than the turbid gel (Fig. S1, ESI $\dagger$ ). Scanning electron microscope (SEM) images showed that the turbid gel contained spherical structures of around $1 \mu \mathrm{m}$ in diameter (Fig. 2a and b), whereas the transparent gel showed a continuous network of fibres (Fig. 2c and d). The decrease in size of structures accounts for the change in turbidity, with the larger structures scattering more light. The spherical structures appear to be formed from bundles of fibres, but it is also possible that these fibres could have been formed as the gel dried. Imaging of the gels using an optical microscope shows that these spherical structures are present in the hydrated gel, but there is insufficient resolution to detect the fibrous structures (Fig. S2, ESI $\dagger$ ). This apparent change in microstructure is highly unusual as there are very few gel-to-gel transitions reported. Furthermore, the change from larger to smaller structures would seem to be energetically unfavourable.

The evolution of the gel network was monitored after the addition of GdL by measuring the rheological properties, the change in turbidity and by recording the $\mathrm{pH}$ over time (Fig. 3). The change in turbidity (strictly we have measured the change in absorbance at $600 \mathrm{~nm}$ as a proxy for turbidity) after an hour indicates that self-assembly began before the $\mathrm{pH}$ had reached the apparent $\mathrm{p} K_{\mathrm{a}}$ of the molecule (the apparent $\mathrm{p} K_{\mathrm{a}}$ was determined from the plateau in the $\mathrm{pH}$ titration data as we have described elsewhere $^{23}$ ). For related LMWGs, we have shown that assembly occurs at the apparent $\mathrm{p} K_{\mathrm{a}}$ of the gelator, with gelation generally beginning at a $\mathrm{pH}$ at or just below this $\mathrm{p} K_{\mathrm{a}}{ }^{22-24}$

Here, the increase in turbidity occurred before any gelation occurred, as shown by the rheological data (Fig. 3). As expected from our previous work, the storage modulus $\left(G^{\prime}\right)$ and the loss modulus $\left(G^{\prime \prime}\right)$ began to increase at the apparent $\mathrm{p} K_{\mathrm{a} \cdot}{ }^{23}$ The gel developed by a two-stage process as we have observed many times before. $^{23,25}$ Interestingly however, the gel became transparent after the $\mathrm{pH}$ had equilibrated, and the rheological properties

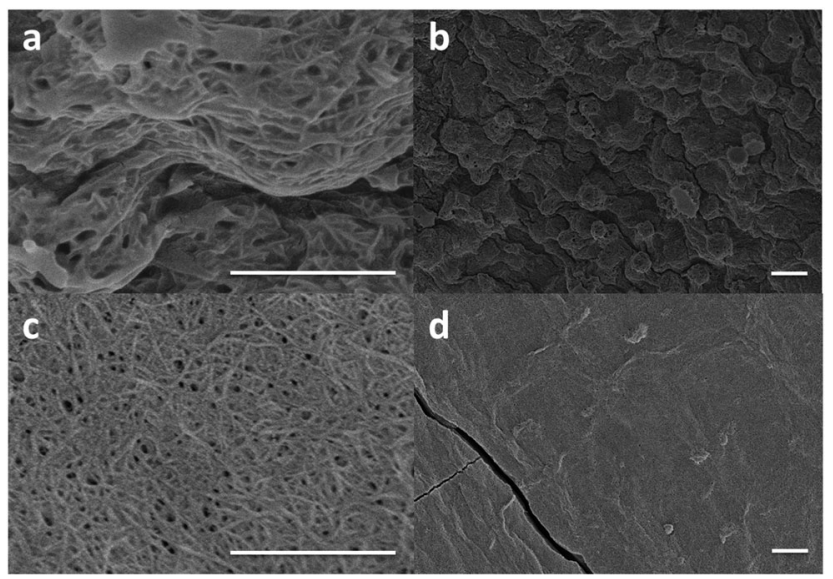

Fig. 2 (a) and (b) SEM images of the turbid gel. (c) and (d) SEM images of the transparent gel. In (a) and (c), the scale bar represents $1 \mu \mathrm{m}$. In (b) and (d) the scale bar represents $2 \mu \mathrm{m}$.

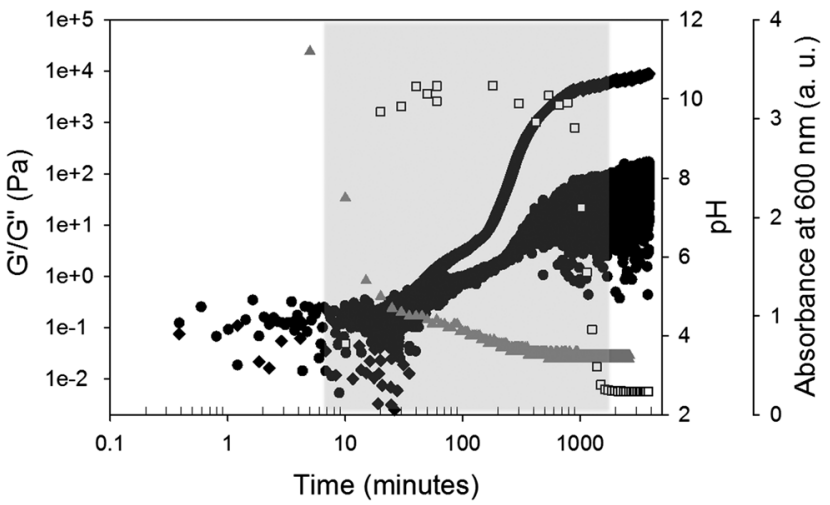

Fig. 3 Graph showing the evolution of the gel network. The diamonds represent the storage modulus, circles represent the loss modulus. $\mathrm{pH}$ is represented by grey triangles. The change in absorbance at $600 \mathrm{~nm}$ (turbidity) is represented by open squares. The grey area indicates that the solution or gel is optically turbid.

continued to increase over an extended period. These results suggest that the changes observed in the gel network at longer times are not simply a result of changes in the $\mathrm{pH}$ of the bulk gel.

The higher the surface area of the air-water interface, the quicker the gel became transparent (Fig. S3, ESI $\dagger$ ). Additionally, the rate of change was affected by the concentration of gelator used (Fig. S4, ESI $\dagger$ ). This suggests that the change in gel structure is influenced by air, presumably due to an atmospheric gas such as carbon dioxide or oxygen. This was further confirmed by degassing a solution of gelator and adding it to a pre-weighed amount of GdL under a nitrogen atmosphere. The gel still underwent a turbid to transparent transition, but it took much longer for the gel to become completely transparent compared to a gelation exposed to air (Fig. S5 and S6, ESI †). For these experiments in a sample tubes and cuvettes suitable for our forms of analysis, complete sealing and exclusion of atmospheric gases was difficult. When we carried out the gelation under strict conditions, no decrease in turbidity was observed over 4 days (Fig. S7, ESI $\dagger$ ).

The same process was then carried out under an enriched carbon dioxide atmosphere. This gel showed a much quicker change from turbid to transparent compared to the gel exposed to air (Fig. S8, ESI $\dagger$ ). This indicates that the gel aging is influenced by the diffusion of $\mathrm{CO}_{2}$ into the solution, presumably forming carbonates. This carbonate formation could either subtly change the $\mathrm{pH}$, which could cause a rearrangement in the gel network, or the carbonate may change the salt concentration, which could also cause a change within the gel structure. To see whether a local pH change was causing the transition, an acid-sensitive pH indicator, bromophenol blue, was added during the gelling process. The $\mathrm{pH}$ indicator showed no difference in $\mathrm{pH}$ at both the transparent and turbid part of the gel (Fig. S9, ESI $\dagger$ ). To investigate whether carbonate concentration was the cause of the gel aging, the gelator was dissolved at high $\mathrm{pH}$ using potassium carbonate instead of sodium hydroxide and again gelled with GdL. The gel went through the same turbidity and gelation 
process as with the sodium hydroxide, however the length of time the gel was in the turbid phase was much shorter (Fig. S10 and S11, ESI $\dagger$ ). The $\mathrm{pH}$ change and rheology of this gel was also recorded and showed similar observations to the sodium hydroxide prepared gels (Fig. S12, ESI $\dagger$ ). Self-assembly took longer to begin due to a slower drop in $\mathrm{pH}$ resulting from the use potassium carbonate (as expected, the rate of GdL hydrolysis is dependent on the salt concentration). ${ }^{21}$ To rule out a cation effect, the gelation was carried out using potassium hydroxide to dissolve the LMWG. Here, the rheological and turbidity data matched that for sodium hydroxide, showing that the differences are due to the carbonate rather than the potassium ions (Fig. S13, ESI $\dagger$ ). Preparing gelator solutions with potassium bicarbonate again resulted in an increase in the rate at which the gel changed from turbid to transparent compared to the solutions prepared with sodium hydroxide.

This LMWG therefore shows an extremely unusual aging profile. As noted above, discussion of aging of such gels is rarely discussed. Shi et al. observed a phenylalanine based gelator that underwent a turbid to transparent transition. ${ }^{16}$ This transition was attributed to a reversible molecular rearrangement of the gelator molecules. Considering the hydrophobicity of the LMWG used here, we expect that the solubility in water is very low at the final $\mathrm{pH}$ of the gel. Hence, it is unlikely that the gel is changing due to dissolution of the LMWG. Instead, we hypothesise that subtle re-arrangements are occurring that lead to the transition from the turbid gel to the transparent gel. Also, after freeze-drying and dissolving in deuterated DMSO, the NMR spectrum of both the turbid and transparent gels showed no change to the gelator (Fig. S14 and S15, ESI $\dagger$ ), as did mass spectrometry (Fig. S16 and S17, ESI $\dagger$ ). Infrared spectroscopy of the turbid and transparent gels also showed no change in packing on the molecular level (Fig. S18, ESI $\dagger$ ) suggesting any transition observed in the gel must be due to a purely morphological rearrangement of the fibres and not a chemical change on the molecular scale.

Perhaps the closest comparison to our system is that reported by Mallia et al., where opaque to transparent gel-to-gel transitions were observed for $\mathrm{CCl}_{4}$ organogels. ${ }^{26}$ The transitions were ascribed to the reversible inclusion of further solvent molecules between the fibres, resulting in a decrease in turbidity, as the opaque gels were heated. Another example of an opaque to transparent transition was shown by Saha et al. this process was reversible with heat cycling but the opaque gel showed weaker rheological properties than the transparent gel. ${ }^{27}$ A number of LMWG are known to be salt sensitive. For example, Nebot et al. have shown that there can be changes in turbidity when specific anions are added to the top of pre-formed gels, which was related to changes in the solubility of the LMWG via the Hofmeister series, ${ }^{28}$ which again could lead to changes in the hydrophilicity of the gelator and hence the amount of included/ bound water. We hypothesise that the addition of carbonate affects the hydrophilicity of the fibres, reducing the inter-fibre interactions and changing the amount of bound water molecules. This leads to a reduction in the highly scattering aggregated fibres and hence a reduction in turbidity.

In summary, the change in turbidity for the gels reported here appear to be driven by a rearrangement of fibres in the gel from large spherical structures to randomly orientated fibrous network. This gel-to-gel transition occurs due to an increased carbonate concentration as a result of $\mathrm{CO}_{2}$ in the air dissolving into solution. This rate of process could be increased by adding carbonate into the solution prior to gelation. We stress that this is very different to the $\mathrm{CO}_{2}$-triggered gelation reported elsewhere, ${ }^{29}$ since gelation occurs under nitrogen; rather it is the gel-to-gel transition that seems to be triggered by the presence of carbonate. These results highlight the importance in gel aging for such systems. Other systems may also go through less visually obvious gel-to-gel transitions that may affect the properties of the gel, something which is important when gels are used for specific applications.

ED thanks the EPSRC for a DTA studentship. DA thanks the EPSRC for a Fellowship (EP/L021978/1).

\section{Notes and references}

1 K. J. Skilling, F. Citossi, T. D. Bradshaw, M. Ashford, B. Kellam and M. Marlow, Soft Matter, 2014, 10, 237-256.

2 V. B. Rajendra, P. P. Kalshetti, D. N. Dixit and P. P. Parekh, Int. J. Pharm. Pharm. Sci., 2012, 4, 1-7.

3 C. Yu, M. Xue, K. Liu, G. Wang and Y. Fang, Langmuir, 2014, 30, 1257-1265.

4 D. Buenger, F. Topuz and J. Groll, Prog. Polym. Sci., 2012, 37, 1678-1719.

5 J. Cui, Z. Shen and X. Wan, Langmuir, 2009, 26, 97-103.

6 K. A. Houton, K. L. Morris, L. Chen, M. Schmidtmann, J. T. A. Jones, L. C. Serpell, G. O. Lloyd and D. J. Adams, Langmuir, 2012, 28, 9797-9806.

7 J. H. van Esch, Langmuir, 2009, 25, 8392-8394.

8 J. Raeburn, A. Zamith Cardoso and D. J. Adams, Chem. Soc. Rev., 2013, 42, 5143-5156.

9 J. R. Moffat and D. K. Smith, Chem. Commun., 2008, 2248-2250.

10 Y. Wang, L. Tang and J. Yu, Cryst. Growth Des., 2008, 8, 884-889.

11 L. Yan, Y. Xue, G. Gao, J. Lan, F. Yang, X. Su and J. You, Chem. - Eur. J., 2010, 16, 2250-2257.

12 M. Lescanne, P. Grondin, A. d'Aléo, F. Fages, J. L. Pozzo, O. M. Monval, P. Reinheimer and A. Colin, Langmuir, 2004, 20, 3032-3041.

13 S. A. Jamieson, K. W. K. Tong, W. A. Hamilton, L. He, M. James and P. Thordarson, Langmuir, 2014, 30, 13987-13993.

14 R. G. Weiss, J. Am. Chem. Soc., 2014, 136, 7519-7530.

15 M. M. Smith and D. K. Smith, Soft Matter, 2011, 7, 4856-4860.

16 J. Shi, Y. Gao, Z. Yang and B. Xu, Beilstein J. Org. Chem., 2011, 7, 167-172.

17 F. Rodriguez-Llansola, J. F. Miravet and B. Escuder, Chem. Commun., 2009, 209-211.

18 C. Yan and D. J. Pochan, Chem. Soc. Rev., 2010, 39, 3528-3540.

19 A. Aufderhorst-Roberts, W. J. Frith, M. Kirkland and A. M. Donald, Langmuir, 2014, 30, 4483-4492.

20 D. J. Adams, M. F. Butler, W. J. Frith, M. Kirkland, L. Mullen and P. Sanderson, Soft Matter, 2009, 5, 1856-1862.

21 Y. Pocker and E. Green, J. Am. Chem. Soc., 1973, 95, 113-119.

22 L. Chen, S. Revel, K. Morris, L. C. Serpell and D. J. Adams, Langmuir, 2010, 26, 13466-13471.

23 L. Chen, K. Morris, A. Laybourn, D. Elias, M. R. Hicks, A. Rodger, L. Serpell and D. J. Adams, Langmuir, 2010, 26, 5232-5242.

24 K. L. Morris, L. Chen, J. Raeburn, O. R. Sellick, P. Cotanda, A. Paul, P. C. Griffiths, S. M. King, R. K. O'Reilly, L. C. Serpell and D. J. Adams, Nat. Commun., 2013, 4, 1480.

25 C. Colquhoun, E. R. Draper, E. G. B. Eden, B. N. Cattoz, K. L. Morris, L. Chen, T. O. McDonald, A. E. Terry, P. C. Griffiths, L. C. Serpell and D. J. Adams, Nanoscale, 2014, 6, 13719-13725.

26 V. A. Mallia, P. D. Butler, B. Sarkar, K. T. Holman and R. G. Weiss, J. Am. Chem. Soc., 2011, 133, 15045-15054.

27 S. Saha, J. Bachl, T. Kundu, D. D. Diaz and R. Banerjee, Chem. Commun., 2014, 50, 7032-7035.

28 V. J. Nebot, J. J. Ojeda-Flores, J. Smets, S. Fernández-Prieto, B. Escuder and J. F. Miravet, Chem. - Eur. J., 2014, 20, 14465-14472. 29 M. George and R. G. Weiss, J. Am. Chem. Soc., 2001, 123, 10393-10394. 